\title{
The spectrum of histomorphological features in psoriasis: a three years study
}

\author{
Arora D. ${ }^{1}$, Mittal A. ${ }^{2}$, Ahmad F. ${ }^{3}$, Dutta S. ${ }^{4}$, Awasthi S. ${ }^{5}$ \\ ${ }^{1}$ Dr. Deepti Arora, Assistant Professor, ${ }^{2}$ Dr. Ankita Mittal, Assistant Professor, ${ }^{3}$ Dr. Faiyaz Ahmad, Associate Professor, \\ ${ }^{4}$ Dr. Shyamoli Dutta, Professor, ${ }^{5}$ Dr. Seema Awasthi, Professor, all authors are affiliated with Department of Pathology, \\ Teerthankar Mahaveer Medical College \& Research Centre (TMMC \& RC), Bagadpur, Moradabad, Uttar Pradesh, India.
}

Corresponding Author: Dr. Deepti Arora, Assistant Professor, Ankita Mittal, Assistant Professor, Department of Pathology, Teerthankar Mahaveer Medical College \& Research Centre (TMMC \& RC), Bagadpur, Moradabad, Uttar Pradesh, India., Email: deepti.a15@gmail.com

\begin{abstract}
Introduction: Psoriasis is a common relapsing chronic inflammatory dermatological disease associated with significant morbidity. Psoriasis is the prototype of a group of cutaneous disorders (psoriasiform dermatitides). Although histopathology is considered 'gold standard' for the diagnosis of psoriasis, at times even histopathological findings are confusing and inconclusive. Aim of the study: To study the histopathological findings at different stages of psoriasis and its subtypes. Materials and Methods: A total of 101 cases were studied for histopathological features. Results: Most of the cases were noted in the age group of 31 to 40 years $(25.9 \%)$. There was a male predominance with male to female ratio of 2.7:1. Psoriasis vulgaris was the most common clinical type followed by Palmoplantar psoriasisand Erythrodermic psoriasis. Parakeratosis was the only consistent feature followed by ddilated blood vessels/abnormal capillary pattern and elongation of rete ridges with thickening at lower ends. The other frequent features were acanthosis and agranulosis / hypogranulosis. Conclusion: Histopathology of psoriatic plaques exhibits heterogeneity and microscopic features will vary according to the stage of the disease. Though most of the predominant morphologic features of psoriasis are related to the epidermis, the presence of dilated blood vessels and/or abnormal capillary pattern is a constant finding in all stages of psoriasis and thus, can help in diagnosis of psoriatic lesion when in doubt.
\end{abstract}

Key words: Psoriasis, Parakeratosis, Acanthosis

\section{Introduction}

Psoriasis is a common relapsing chronic inflammatory dermatological disease associated with significant morbidity [1-3]. It is characterized by sharply demarcated, erythematous papules and plaques with abundant silvery white-scales [4]. Psoriasis is universal in occurrence affecting $1 \%$ to $3 \%$ of population worldwide [2,5]. There is paucity of data related to exact burden of psoriasis in India. However, in few separate hospital-based studies the incidence of psoriasis is reported as $0.44 \%-2 \%$ and $2.3 \%[2,6]$.

Psoriasis is the prototype of a group of cutaneous disorders (psoriasiform dermatitides) that shows psoriasiform epidermal hyperplasia, defined as regular elongation of the rete ridges with preservation of the rete ridge-dermal papillae pattern [7,8]. Psoriasis has different clinical subtypes that may simulate various

Manuscript received: $18^{\text {th }}$ January 2019

Reviewed: $28^{\text {th }}$ January 2019

Author Corrected: $6^{\text {th }}$ February 2019

Accepted for Publication: $11^{\text {th }}$ February 2019 other dermatological disorders. This presents a diagnostic challenge for the clinician and histopathological confirmation becomes mandatory [3]. The histologic diagnosis depends upon an aggregate of histologic criteria, some characteristic of psoriasis and others shared with other dermatoses [9]. The microscopic picture of psoriasis varies with the evolutionary stage of psoriatic lesion [10]. Although the histopathology is considered 'gold standard' for the diagnosis of psoriasis, at times even histopathological findings are confusing and inconclusive [3].

Aim of The Study- In this study, an attempt was made to establish profile of histopathological findings at different stages of psoriasis and its subtypes.

\section{Materials and Methods}

No ethical issues were involved in this study. This was aprospective; hospital-based, cross-sectional studyand was carried out in the Department of Pathology of a 


\section{Original Research Article}

tertiary care medical institute in India for a period of three years. The study material constituted biopsy samples from patients of all age groups attending dermatology outpatient department (OPD) with the clinical diagnosis of psoriasis or with psoriasis as one of the differential diagnosis. Skin biopsies either as punch biopsy or incisional biopsy was done by the dermatologist as an out-patient procedure in the dermatology OPD.

The tissue specimen was immediately put in $10 \%$ buffered neutral formalin for fixation and was sent to the department of pathology. In the histopathology section, these specimens were fixed adequately and were submitted for routine histopathological processing. This was followed by cutting the sections at five-micron thickness and staining with routine hematoxylin and eosin stains. All the stained sections were observed under light microscope and the histopathological findings were noted.

\section{Inclusion criteria}

1. Clinically suspected cases of psoriasis

2. Both genders and all age groups were included

\section{Exclusion criteria}

1. Inadequate biopsy samples (less than $4 \mathrm{~mm}$ ) and biopsies showing only epidermis or dermis on histologic examination were excluded.

2. Skin biopsy done for cases other than psoriasis or suspected psoriasis

3. Repeat skin biopsies within the study period

No scoring system was used for this study. For statistical analysis, the percentages were done.

\section{Results}

Total of 101 biopsy samples from patients were evaluated for histological features of psoriasis.Most of the cases were noted in the age group of 31 to 40 years $(25.9 \%)$ followed closely by age group of 41 to 50 years $(23.8 \%)$. Only one case was noted in 0 to 10 years age group $(0.9 \%)$. The youngest patient was 6 years old and oldest was 89 years old at the time of biopsy.

The study showed male predominance with male to female ratio of 2.7:1. (74 males and 27 females). Psoriasis vulgaris was the most common clinical type (89.3\%) followed by Palmoplantar psoriasis $(6.9 \%)$ and Erythrodermic psoriasis $(2.9 \%)$ in the present study. Inverse psoriasis was the least common $(0.9 \%)$ clinical type.

Among all histologic features observed, Parakeratosis was the only feature which was seen in all biopsy samples. Dilated blood vessels/abnormal capillary pattern (98.1\%) and elongation of rete ridges with thickening at lower ends (98.1\%) were second most common histologic feature after parakeratosis. The other frequent features were acanthosis (96.1\%), pallor of upper layer of epidermis (88.1\%), and agranulosis/hypogranulosis (83.1\%).

Kogoj spongiform pustules were the least common histologic feature and were identified in only $9(8.9 \%)$ out of 101 biopsy samples. The presence of spongiosis (10.8\%) and exocytosis of neutrophils (20.7\%) were also infrequent histopathological finding in biopsy samples of psoriatic patients. Histological features observed in the biopsy samples are summarized in table.1.

Table-1: Distribution of histologic features present in biopsy samples.

\begin{tabular}{|l|c|c|}
\hline Histological features & No. of cases & Percentage (\%) \\
\hline Parakeratosis & 101 & $100 \%$ \\
\hline Hyperkeratosis & 45 & $44.5 \%$ \\
\hline Acanthosis (regular epidermal hyperplasia) & 97 & $96.1 \%$ \\
\hline Elongation of rete ridges with thickening at lower ends & 99 & $98.1 \%$ \\
\hline Suprapapillary thinning & 42 & $41.5 \%$ \\
\hline Agranulosis/ hypogranulosis & 84 & $83.1 \%$ \\
\hline Spongiosis & 11 & $10.8 \%$ \\
\hline Munro microabscess & 30 & $29.7 \%$ \\
\hline Kogojspongioform pustule & 9 & $8.9 \%$ \\
\hline Exocytosis of neutrophils & 21 & $20.7 \%$ \\
\hline
\end{tabular}




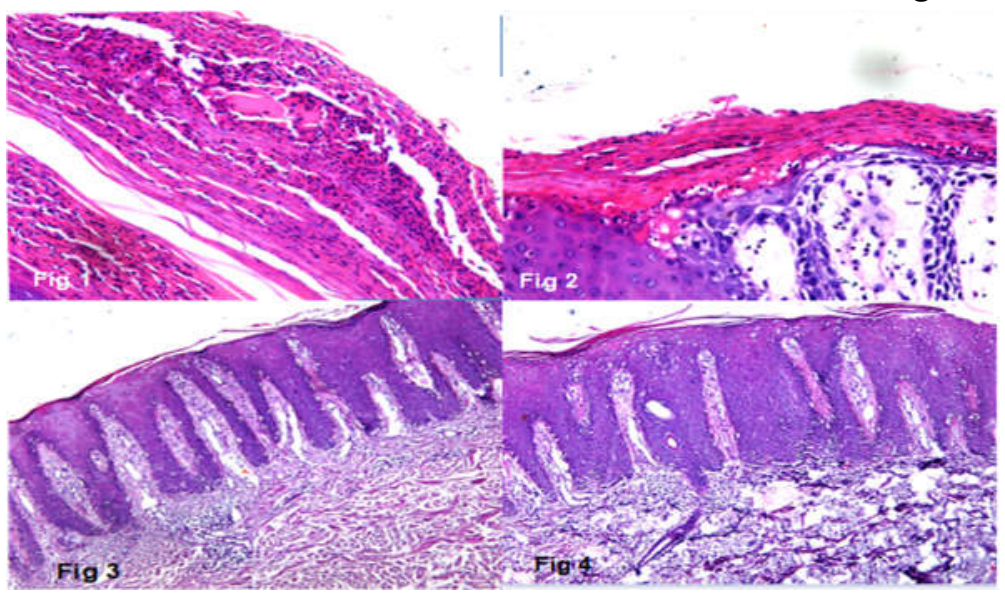

Fig 1: Munro micro abscesses (H \& E Section, 10X)

Fig 2: Supra-papillary thinning with prominent blood vessels (H \& E Section, 10X)

Fig 3: Shows elongation of rete ridges with thickening at lower ends (H \& E Section, 10X)

Fig 4: Acanthotic epithelium (H \& E Section, 10X)

\section{Discussion}

Psoriasis is a genetically determined erythematosquamous dermatitis characterized by abnormal keratinocyte proliferation resulting in thickening of epidermis [8]. In its classic presentation, the disease comprises of well circumscribed reddish scaly papules and plaques typically on the elbow, knee and scalp, in addition to other cutaneous sites [11]. In this typical form, the diagnosis is straightforward based on clinical features alone and skin biopsy for histopathological examination is seldom required [8].

However, histologic analysis of skin biopsies can be helpful to confirm the diagnosis of psoriasis in its classic form and clinically atypical variants [11]. With this background, the present study was undertaken and biopsy samples of 101 psoriatic patients were systematically evaluated on histopathology for establishing the morphological features of psoriasis.

In our study, psoriasis vulgaris was the most common clinical type which accounted for $89.3 \%$ cases followed by palmoplantar psoriasis (6.9\%) and erythrodermic psoriasis $(2.9 \%)$. The inverse psoriasis was the least common $(0.9 \%)$. Similar observations were found by other authors who reported maximum cases of psoriasis vulgaris ( $\geq 85 \%$ ) and almost similar number of cases of erythrodermic psoriasis $(2-3 \%)$ and inverse psoriasis (1-2\%) [12-15]. Thomas $\mathrm{J}$ et al reported higher incidence of palmoplantar psoriasis followed by palmoplantar psoriasis constituting $45 \%$ cases and $44 \%$ cases of psoriasis vulgaris [16]. Puri $\mathrm{N}$ et al found marginally higher cases of psoriasis vulgaris $(32 \%)$ than guttate psoriasis $(28 \%)$ followed by erythrodermic psoriasis $(16 \%)$ and similar incidence of generalized pustular and palmoplantar psoriasis (12\%) [17].
Allthe 101 biopsies were examined for the various morphological features described in literature for psoriasis in the present study. Parakeratosis was the consistent histopathological feature which was noted in all patients. Otherauthorsin their respective studies have also described parakeratosis as the most consistent histopathological feature of psoriasis $[8,11,15,18-20]$. However, Mehta $\mathrm{S}$ et aland Puri $\mathrm{N}$ et al found parakeratosis in $52 \%$ and $56 \%$ cases respectively $[3,17]$. Hyperkeratosis was observed in $44.5 \%$ biopsies of psoriatic patients; the findings are comparable to the study by Puri $\mathrm{N}$ et al [17].They observed hyperkeratosis in $64 \%$ cases. However, studies by Raghuveer $\mathrm{C}$ et al and Abdu NN et al reported hyperkeratosis in $89 \%$ and $88 \%$ cases respectively [14,21]. Bai et al, Chandanwale $\mathrm{SS}$ et al and Park JH et al found hyperkeratosis in all the biopsies of psoriasis patient $[15,19,20]$.

In the present study, acanthosis (regular epidermal hyperplasia) was present in $96.1 \%$ of biopsies. Our results are in concordance with other studies in which acanthosis was observed in about $75 \%$ or more biopsies $[3,14,17,19,22]$. However, Park JH et al reported lesser prevalence of this feature (46.7\%) as compared to other authors as well as the present study [20]. Elongation of rete ridges with thickening at the lower ends or 'club shaped' ridges was noticed in $98.1 \%$ biopsy samples. Our results are comparable with the studies conducted by Park JH et al and Kim BY et al $[20,22]$. Kim BY et al noticed elongation of rete ridges in $93 \%$ cases while Park JH et al reported thickening of lower ends in $100 \%$ biopsy samples. However few studies reported elongation of rete ridges in only $66-76 \%$ cases $[14,17,21]$. In a study conducted by Chandanwale SS et al they reported that slender long rete pegs favor the 
Original Research Article

histopathological diagnosis of psoriasis which was not a consistent finding in our study [19]. In the present study, suprapapillary thinning was noted in $41.5 \%$ biopsy samples, findings similar to the studies conducted by Mehta $\mathrm{S}$ et al and Puri $\mathrm{N}$ et al [3,17].This finding is in contrast to other studies which reported variable prevalence of this feature $[15,20,22]$. Ghasmeni et al showed thinning of suprapapillary plate to be significantly more common in psoriasis [23]. We have observed the feature of elongation of rete ridges with thickening at lower ends in almost all biopsies but the suprapapillary thinning was observed in only $41.5 \%$ cases. The suprapapillary epidermal thinning is a relative observation on comparison with markedly elongated rete ridges and therefore may not be observed in all cases of psoriasis.

Trozak explained the importance of club shaped rete ridges as characteristic of epidermis in psoriasis which helps in differentiating the disease from Psoriasiform dermatitis. He also included this feature in histological grading system for psoriasis developed by him [9]. Our findings also re-emphasize the club shaped rete ridges as characteristic of psoriasis even if not associated with suprapapillary thinning. In the present study, agranulosis/ hypogranulosis was noted in $83.1 \%$ biopsy samples. Similar results have been observed in previous studies which reported presence of agranulosis/ hypogranulosis in $68 \%-100 \%$ cases. In contrast, a study conducted by Chandanwale SS et al found agranulosis in $32 \%$ cases [19]. The cause for absent granular layer or decreased/hypo granular layer is not known. However, it seems that this is probably related to epidermal hyperproliferation in earlier stage of disease, as later lesions of psoriasis may show intact granular layer $[24,25]$.

In our study the spongiosis was present in $10.8 \%$ biopsies.Mehta $\mathrm{S}$ et al and Puri $\mathrm{N}$ et al found spongiosis in around $40 \%$ cases $[3,17]$. Bai $\mathrm{S}$ et al observed spongiosis in $91.7 \%$ [15]. Park $\mathrm{JH}$ et al noticed slight spongiosis in all the cases in their respective studies [20]. Thus, our results are in contrast to these studies. Munro microabscess was observed in $29.7 \%$ biopsies. Other studies observed Munro microabscess in approximately $50 \%-60 \%$ biopsies $[3,14,15,19,21,22]$. A study done by Park JH et al found this feature in almost all biopsies [20]. Kogoj spongiform pustules were seen in $8.9 \%$ biopsy samples. It was found that though Munro microabscess and Kogoj spongiform pustules are important diagnostic features of psoriasis, they are not always present on histopathology $[1,9,18,26]$. In the present study, dilated blood vessels/abnormal capillary pattern in dermal papillae were seen in $98.1 \%$ of biopsy samples. Ourfindings are in concordance with the studies by Raghuveer $\mathrm{C}$ et al, Chandanwale et al and Abdu NN et al [14, 19,21] They reported dilated blood vessels in dermal papillae in $98 \%$ and $100 \%$ and $90 \%$ cases respectively. The studiesby Mehta $\mathrm{S}$ et al and Puri $\mathrm{N}$ et al reported this feature in $80 \%$ and $54 \%$ cases respectively [3,17]. We have also observed that the dilated blood vessels/abnormal capillary pattern is seen in almost all cases of psoriasis indicating that in all stages of psoriasis, this is a consistent finding.

We observed exocytosis of neutrophils in $20.7 \%$ biopsy samples. Mehta $\mathrm{S}$ et alfound this histologic feature in all biopsy samples, whereas, Puri $\mathrm{N}$ et al found exocytosis of neutrophils in $48 \%$ biopsies [3,17]. Our findings are in sharp contrast with the above studies. Griffin TD et al described exocytosis of neutrophils as a histopathological feature of acute psoriasis while chronic psoriatic plaques lack the presence of neutrophils [27]. The finding of neutrophil exocytosis appears to be a variable finding and may be limited to early or late stage. It may be seen as diagnostic clue or it may be of little help. We also did not find this finding in large number of biopsy specimens in our study.

\section{Conclusions}

Histopathology of psoriatic plaques though show some consistent features but will always exhibit heterogeneity and microscopic features will vary according to the stage of the disease. Most of the predominant morphologic features of psoriasis are related to the epidermis, with varying histopathological findings.

However, the presence of dilated blood vessels and/or abnormal capillary pattern is a constant finding in all stages of psoriasis. It is recommended to specifically look for these findings in cases of biopsies suspected of psoriasis as their presence can help in diagnosis of psoriatic lesions when in doubt.

Findings: Nil; Conflict of Interest: None initiated Permission from IRB: Yes

\section{References}

1. Altman EM, Kamino H. Diagnosis: psoriasis or not? What are the clues? SeminCutan Med Surg. 1999 Mar; 18 (1):25-35.

2. Dogra S, Yadav S. Psoriasis in India: prevalence and pattern. Indian J Dermatol VenereolLeprol. 2010 NovDec; 76 (6):595-601. doi: 10.4103/0378-6323.72443.

3. Mehta S, Singal A, Singh N, et al. A study of clinic histopathological correlation in patients of psoriasis and psoriasiform dermatitis. Indian J Dermatol Venereol Leprol. 2009 Jan-Feb;75(1):100. 


\section{Original Research Article}

4. Khalil FK, Keehn CA, Saeed S, et al. Verrucous psoriasis: a distinctive clinicopathologic variant of psoriasis. Am J Dermatopathol. 2005 Jun;27(3):204-7.

5. Linden KG, Weinstein GD. Psoriasis: current perspectives with an emphasis on treatment. Am J Med. 1999 Dec;107(6):595-605.

6. Kaur I, Handa S, Kumar B. Natural history of psoriasis: a study from the Indian subcontinent. J Dermatol. 1997 Apr;24(4):230-4.

7. Sehgal VN, Dogra S, Srivastava G, et al. Psoriasiform dermatoses. Indian J Dermatol Venereol Leprol. 2008 Mar-Apr;74(2):94-9.

8. Murphy M, Kerr P, Grant-Kels JM. The histopathologic spectrum of psoriasis. Clin Dermatol. 2007Nov-Dec;25(6):524-8.DOI:10.1016/j.clindermatol. 2007. 08.005

9. Trozak DJ. Histologic grading system for psoriasis vulgaris. Int J Dermatol. 1994 May;33(5):380-1.

10. Micali G, Lacarrubba F, Musumeci ML, et al. Cutaneous vascular patterns in psoriasis. Int J Dermatol. 2010 Mar;49(3):249-56. doi: 10.1111/j.1365-4632. 2009. 04287.x.

11. Cribier BJ. Psoriasis under the microscope. JEADV 2006; 20(Suppl 2):3-9.

12. Asokan N, Prathap P, Ajithkumar K, et al. Pattern of psoriasis in a tertiary care teaching hospital in South India. Indian J Dermatol. 2011 Jan;56(1):118-9. doi: 10.4103/0019-5154.77575.

13. Golpour M, Hosseini SH, Khademloo M, et al. Depression and Anxiety Disorders among Patients with Psoriasis: A Hospital-Based Case-Control Study. Dermatol Res Pract. 2012;2012:381905. doi: 10.1155/ 2012/ 381905. Epub 2012 Jul 16.

14. Raghuveer C, Shivanand DR, Rajashekar N. Clinico-histopathological Study of Psoriasis. Int J Sci Stud 2015;3(7):176-179.

15. Bai S, Sowmya S. Histopathologic diagnostic parameters of psoriasis; a clinicopathological study. Int J Res Med Sci 2016;4:1915-20.

16. Thomas J, Kumar NA, Manoharan D, Cynthia S, Prabu SKS, Ahmed NA. A study of comorbid conditions in psoriasis. Journal of Pakistan Association of Dermatologists 2009;19:200-2.
17. Puri N, Mahajan BB, Kaur S. Clinicohistopathological Correlation of Psoriasis in Acute Exacerbation. 2012;1:455. doi:10.4172/scientificreports. 455

18. De Rosa G, Mignogna C. The histopathology of psoriasis. Reumatismo. 2007;59 Suppl 1:46-8.

19. Chanadanwale SS, Panicker NK, Kulkarni SP, Shah KR, Kumar H, Sharma YK, et al. Morphometry analysis of psoriasis and psoriasiform dermatitis: A retrospective study of 50 cases. Med J DY Patil Univ 2015;8:43-7.

20. Park JH, Park YJ, Kim SK, et al. Histopathological Differential Diagnosis of Psoriasis and Seborrheic Dermatitis of the Scalp. Ann Dermatol. 2016 Aug;28 (4): 427-32. doi: 10.5021/ad.2016.28.4.427. Epub 2016 Jul 26.

21. Abdu NN, Bugude G, Mallikarjun M, Deepadarshan K. Clinical and histopathological study of psoriasis. Med Pulse International Journal of Medicine 2017;4(2): 58-63.

22. Kim BY, Choi JW, Kim BR, et al. Histopathological findings are associated with the clinical types of psoriasis but not with the corresponding lesional psoriasis severity index. Ann Dermatol. 2015 Feb;27(1):26-31. doi: 10.5021/ad.2015. 27.1. 26. Epub 2015 Feb 3.

23. GhasemiBasir HR, Alirezaei P, Hamian Z, et al. Are quantitative histopathologic criteria capable of differentiating psoriasis from chronic dermatitis? Clin CosmetInvestig Dermatol. 2018 May 10;11:239244. doi: 10.2147/CCID.S160697. eCollection 2018.

24. Barr RJ, Young EM Jr. Psoriasiform and related papulosquamous disorders. J CutanPathol. 1985 Oct; 12 (5): 412-25.

26. Chowaniec O, Jabłońska S, Beutner EH, et al. Earliest clinical and histological changes in psoriasis. Dermatologica. 1981;163(1):42-51.

26. Toussaint S, kamino $\mathrm{H}$. Non- infectious erythematous papular and squamous diseases of the skin. In: Elder D, Elenitsas R, Jaworsky C, Johnson B, editors. Lever's Histopathology of the skin. 8th edition ed. Philadelphia: Lippincott-Raven; 1997. p. 156-63.

27. Griffin TD, Lattanand A, VanScott EJ. Clinical and histologic heterogeneity of psoriatic plaques. Therapeutic relevance.ArchDermatol.1988Feb;124(2):216-20

\section{How to cite this article?}

Arora D, Mittal A, Ahmad F, Dutta S, Awasthi S. The spectrum of histomorphological features in psoriasis: a three years study. Trop J Path Micro 2019;5(2):58-62.doi:10.17511/jopm.2019.i02.02. 